\author{
Military Technical College \\ Kobry El-Kobbah, \\ Cairo, Egypt
}

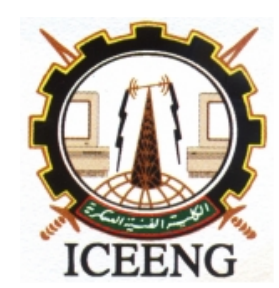

\author{
$7^{\text {th }}$ International Conference \\ on Electrical Engineering \\ ICEENG 2010
}

\title{
AMPLITUDE-ONLY PATTERN SYNTHESIS OF NON-UNIFORM LINEAR ARRAY USING A GENERALIZED PATTERN SEARCH OPTIMIZATION
}

By

Fikret Tokan*
Filiz Gunes*

\section{$\underline{\text { Abstract: }}$}

In this work, synthesis of linear array geometry is first formulated as a linearly constrained multi-objective optimization problem with the goals of minimum sidelobe level, null control and high directivity. Then solved by a Generalized Pattern Search (GPS) algorithm for the optimum element locations and excitation amplitudes. The constraints are imposed on the inter-element spacing and dynamic range ratio of the amplitude tapering to reduce mutual coupling effects between the elements. GPS methods are newly discovered, derivative-free methods where the current iterate is updated by sampling the fitness function at a finite number of points along a suitable set of search directions to find a decrease in the function value. Finally, two worked examples are presented that illustrate the use of GPS synthesis method, and the optimization goal in each example is easily achieved. Furthermore the full-wave simulations of the synthesized arrays are also completed to examine the mutual coupling effects. Finally the results of the GPS algorithm are validated by comparing with results obtained using the genetic algorithm, and the results of the uniform and DolphChebyshev arrays, having the same number of element and the same aperture length.

\section{Keywords:}

Antenna array, directivity, null control, Generalized Pattern Search (GPS) optimization, radiation pattern, sidelobe suppression.

* Yildız Technical University, stanbul, Turkey. 


\section{Introduction:}

In many communication systems one is interested in point-to-point communication, and much more highly directive beam of radiation can be used to advantage. Hence, by arranging elementary radiators into an array, a more directive beam of radiation can be obtained. A more directive beam means that the antenna will also have a higher gain. The other important requirement for a communication system is high ratio of the signal to interference. This is achieved by the suppression of interference and multipath signals and obtaining nulls in the directions of interfering signals. Thus all of these simultaneous requirements necessitate synthesis of an array antenna by a multiobjective optimization process.

In general, these optimization techniques utilize single system variable to be optimized that can be classified into the two categories: One method optimizes the excitation of each element of the uniform array [1-2], and the other adjusts the element locations with uniform excitation [3-4], resulting in a non-uniform array geometry.

In this work, we aimed at the array antenna synthesis by both the linear geometry and excitation amplitudes that result in determination of the physical layout and amplitude tapering network. The objectives of the synthesis process, can briefly be summarized as follows: To maximize beam of the radiation pattern towards the intended user or signal of interest, while obtaining maximum suppression within the required region(s) together with narrow/broad nulls in the directions of interfering signals or signal not of interest. For this purpose all the multiple objectives are simultaneously gathered within a single fitness function as the weighted logarithmic sums of the magnitudes of the array factor and the directivity function. The element location $\left(\mathrm{d}_{\mathrm{n}}\right) \mathrm{s}$ and the excitation amplitude $\left(A_{n}\right) \mathrm{s}, n=1, \ldots . . N$ are chosen as the decision variables to control the far field features in the optimization process.

In the recent years, the evolutionary algorithms such as Genetic Algorithms become popular to synthesize either the physical layout or the feeding network of array antenna. The common characteristics of these techniques are that these are random and nonrepeatable optimization processes.

Here, GPS methods are introduced as an optimization tool into the antenna engineering for the array antenna synthesis. GPS are nonrandom methods for direct searching minima of a function which may be even discontinuous, non-differentiable, stochastic or highly nonlinear [5]. Thus a comparatively fast and repeatable tool is introduced to the array antenna synthesis with no need for gradients of the fitness function.

\section{Problem Formulation of a Non-Uniform Linear Array:}

Fig.1 gives a linear array antenna of isotropic elements distributed symmetrically with 
respect to the origin along the $y$-axis. This linear array antenna has non-uniform interelement spacing and at same time non-uniform excitations, thus the array factor in the azimuth plane can be expressed as follows [6]:

$$
A F=2 \sum_{n=1}^{N} A_{n} \cos \left[\frac{2 \pi d_{n}}{\lambda} \sin \phi+\beta_{n}\right]
$$

where $2 N$ is total number of the antenna elements, $\lambda$ is the wavelength in free space; $A_{n}, \beta_{n}$ are the excitation amplitude and phase of the $n$th element, respectively and $d_{n}$ is the distance from the origin to the $n$th element. In our case, $\beta_{n}$ is fixed zero, thus the broadside arrays are worked out:

$$
A F=2 \sum_{n=1}^{N} A_{n} \cos \left[\frac{2 \pi d_{n}}{\lambda} \sin \phi\right]
$$

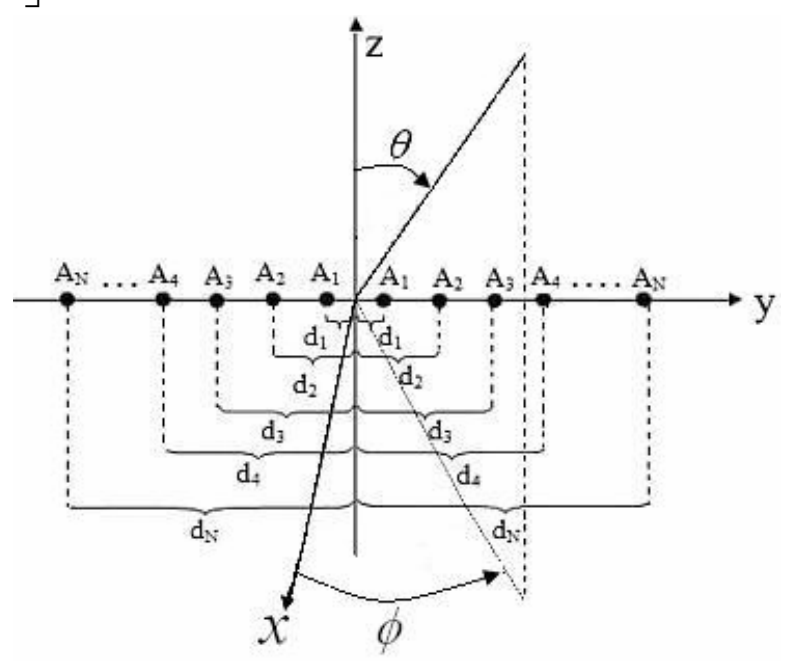

Figure (1): Linear antenna array geometry

In the antenna engineering, measure of the maximizing electromagnetic energy to the intended user is directivity which is defined as [7]:

$$
D=\frac{U}{U_{0}}
$$

where $U$ and $U_{0}$ are the radiation intensities of the directive and isotropic antennas, respectively. Formulation of the directivity is given in details by Stutzman and Thiele [7] for the non-uniformly excited and unequally spaced linear arrays. We adapted this 
formulation to the symmetrical configuration of the linear array antenna of sum pattern in the broadside direction:

$$
D=\frac{2(a)^{2}}{2\left(b_{1}+b_{2}\right)}
$$

where $a=\sum_{n=1}^{N} A_{n}, b_{1}=\sum_{n=1}^{N} \sum_{n=1}^{N} A_{n} A_{m} \frac{\sin \left[2 \pi\left(d_{n}+d_{m}\right)\right]}{2 \pi\left(d_{n}+d_{m}\right)}, b_{2}=\sum_{n=1 n=1}^{N} \sum_{n=1}^{N} A_{n} A_{m} \frac{\sin \left[2 \pi\left(d_{n}-d_{m}\right)\right]}{2 \pi\left(d_{n}-d_{m}\right)}$

Thus, directivity will be considered as an objective in the synthesis process as the measure of focusing the beam to the desired direction as narrow as we require.

\section{GPS Algorithm:}

GPS methods are a class of direct search methods, originally introduced and analyzed by Torczon [5]. A summary of the work on GPS methods can be found in [5]. GPS are the direct methods for searching minima of a function which may be even discontinuous, nondifferentiable, stochastic, or highly nonlinear. As opposed to more traditional optimization methods that use information about the gradient or higher derivatives to search for an optimal point, a GPS algorithm searches a set of points around the current point, looking for one where the value of the fitness function is lower than the value at the current point.

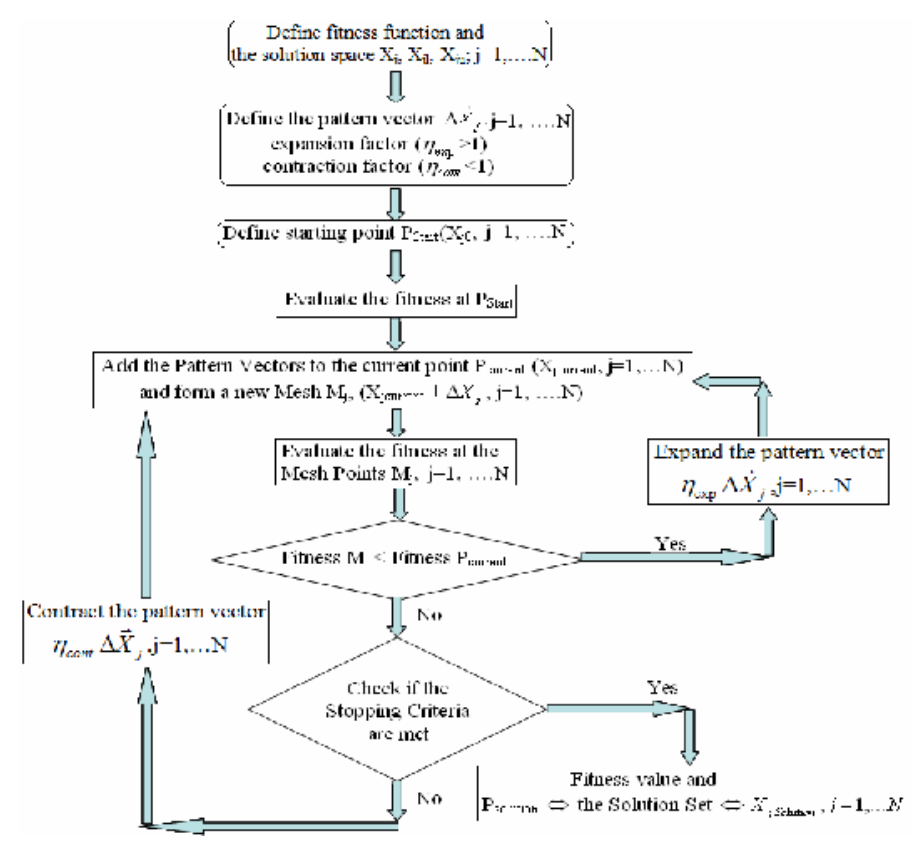

Figure (2): Flow chart for the GPS algorithm 
The GPS algorithm we employed can briefly be explained as follows: It computes a sequence of points closer and closer to the optimal point. At each step, the algorithm searches a set of points, called a mesh, around the current point until finding a point in the mesh where value of the fitness function decreases compared to the value at the current point. This new point becomes the current point at the next step of the algorithm. The mesh is formed by adding a scalar multiple of a fixed set of vectors called "Pattern Vector" to the current point. Flow chart of the GPS algorithm is given in Fig. 2.

\section{Objective Function:}

The GPS presented in the previous section is applied to synthesize array antenna with the linear geometry and excitation amplitudes subject to the radiation pattern requirements. Here, our objectives are to meet the minimum sidelobe level, maximization of the main beam in the desired direction to obtain narrow/ broad nulls in the directions of interfering signals. Thus, these multiple objectives are gathered in a single fitness function as the weighted logarithmic sums of the magnitudes of the array factor, $|A F(\phi)|$ and the directivity D functions given by (3) - (4) as follows:

$$
\begin{aligned}
& \text { Fitness }= w_{1} 20 \log \left\{\sum_{l} \frac{l}{\Delta \phi_{l}} \int_{\phi_{\phi_{l}}}^{\phi_{u l}}|A F(\phi)|\right\}+w_{2} 20 \log \left\{\sum_{i} \max \left\{\mid A F(\phi)_{\phi_{\phi_{i}}}^{\phi_{\phi_{i}}=90^{0}}\right\}\right\} \\
&+w_{3} 20 \log \left\{\sum_{k}\left|A F\left(\phi_{k}\right)\right|\right\}-20 \log D \\
& \text { subject to } d_{n+1}-d_{n} \geq 0.5 \lambda, \quad n=1, \ldots \ldots . . N-1, \\
& \frac{A_{\max }}{A_{\min }} \leq C, \quad C>1
\end{aligned}
$$

In (5), $\phi_{u_{l}}$ and $\phi_{l_{l}}$ are the upper and lower angles of the regions $l=1, \ldots L$ and $\Delta \phi_{l}=\phi_{u_{l}}-\phi_{l_{l}}$, and $\phi_{k}, k=1, \ldots \ldots . . K$, are the directions where the nulls are required, $\phi_{u_{i}}, \phi_{l_{i}}$ are the boundaries of the $i$ th spatial region where the maximum is intended to be suppressed. Besides, $w_{i}, i=1, \ldots 4$ are the weighting coefficients which should be adapted to the problem.

(6.1) and (6.2) give the constraints imposed on inter-element spacing and the dynamic range ratio of the amplitude tapering to reduce mutual coupling effects between the elements of the optimized array antennas. 


\section{Linear Array Antenna Synthesis Examples:}

In this section, synthesis examples of various antenna arrays are worked out to demonstrate the versatilities in performances of the objective function given in (5) subject to the constraints in (6.1) and (6.2). The optimized arrays are compared for directivity and beamwidth with uniform arrays having same aperture length with the arrays obtained using GPS optimizer; while the Dolph-Chebyshev array is synthesized with the same MSLL and aperture length to compare the other radiation pattern properties such as the suppressed regions, null levels and directivities. In order to compare the performance of the GPS optimizer, the same objective function and constraints are applied to the array antenna synthesis by GA optimizer, and then the resulted patterns of both optimizers are compared with each other. Furthermore, fullwave simulations of all the patterns optimized by the GPS are completed using 3D EM field simulator, Computer Simulation Technology (CST) to examine the mutual coupling effects between the antenna elements.

In the first worked example, the sum pattern configuration of a 10-element linear array is synthesized for minimum SLL within the region $\left[14^{\circ}-90^{\circ}\right]$ and obtaining nulls at $40^{\circ}, 50^{\circ}$ and $70^{\circ}$ while maintaining high directivity and narrow beamwidth compared with the counterparts. The corresponding fitness function can be expressed using (5) as:

$$
\begin{aligned}
\text { Fitness } & =20 \log \left\{\max \left\{|A F(\phi)|_{\phi_{l}=14^{\circ}}^{\phi_{\phi}=90^{\circ}}\right\}\right\}-20 \log D \\
& +20 \log \left\{A F\left(40^{\circ}\right) \mid\right\}+20 \log \left\{A F\left(50^{\circ}\right) \mid\right\}+20 \log \left\{A F\left(70^{\circ}\right) \mid\right\}
\end{aligned}
$$

The resulted array pattern from the GPS algorithm is shown in Fig. 3, together with the patterns obtained using uniform array geometry, Dolph-Chebyshev array, GA optimizer and CST simulator. Moreover, the corresponding features of the all patterns are also given in Table 1(a).

It can be seen from Fig. 3 that the uniform array exhibits relatively high SLL. The GA offers an improvement of $4 \mathrm{~dB}$, while the GPS algorithm offers an improvement of $12 \mathrm{~dB}$ in terms of MSLL suppression. It can also be seen from Fig. 3 and Table 1(a) that the aim of obtaining deep nulls at $40^{\circ}, 50^{\circ}$ and $70^{\circ}$ directions has been achieved easily by both the GPS and GA optimizers. Although, Dolph-Chebyshev array is designed for same MSLL with GPS array, relatively deep levels in the directions of interference are achieved using GPS optimizer. This is achieved in exchange for a larger width in the main beam. It can also be said that the radiation pattern of CST simulator is in a good 
agreement with the pattern of GPS array and the levels of at least $-40 \mathrm{~dB}$ in the null desired directions are achieved by the simulator.

The array geometry for this example is shown in Table 1(b). It can be seen from the table that the array length obtained using the GPS algorithm is same as DolphChebyshev and uniform arrays. This is also observed for the rest of the design examples shown next.

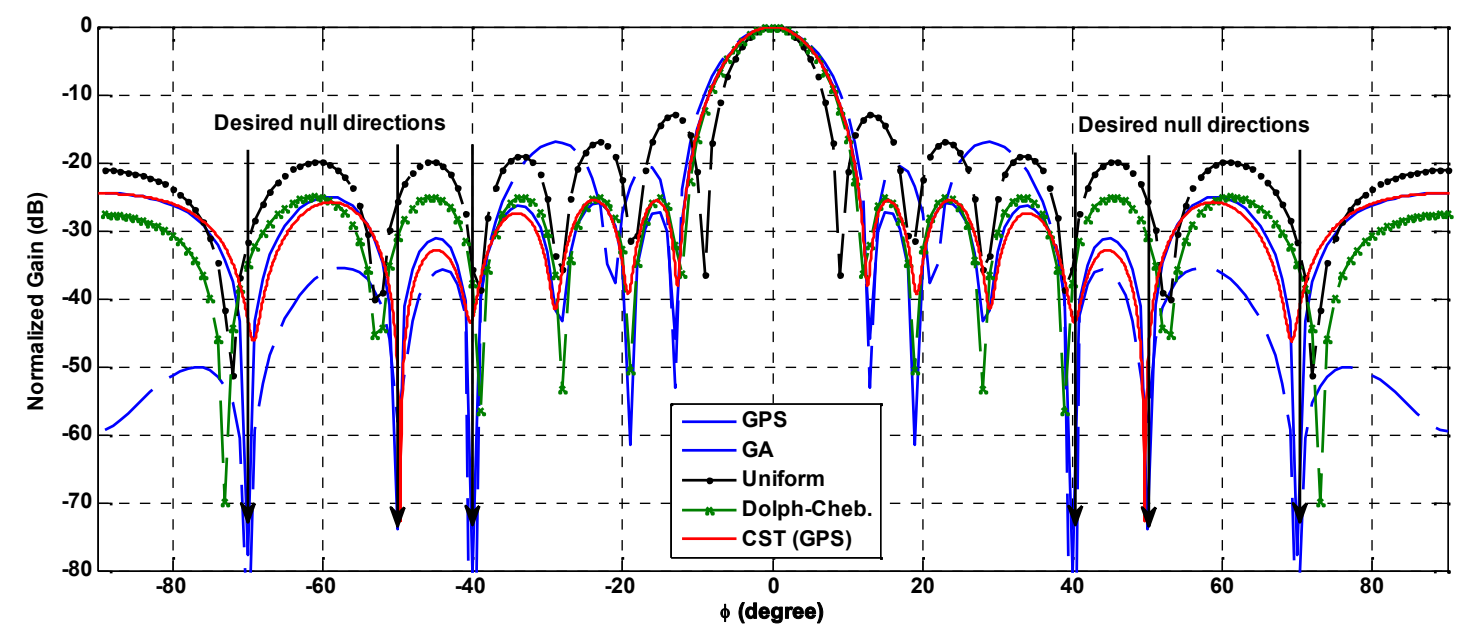

Figure (3): Normalized patterns of the 10-element linear array obtained using five different methods. The region of suppressed SLL is $\left[14^{\circ}, 90^{\circ}\right]$ and nulls are prescribed at: $40^{\circ}, 50^{\circ}, 70^{\circ}$

Table (1): (a) Features (b) Normalized Solution Spaces, of the Radiation Patterns in Fig.5.

\begin{tabular}{|c|c|c|c|c|c|c|}
\hline $\begin{array}{l}\text { Optimization } \\
\text { Algorithm }\end{array}$ & D & $\begin{array}{l}\text { MSLL } \\
(\mathrm{dB})\end{array}$ & $\begin{array}{c}\text { HPBW } \\
\left(\phi_{H P B W}\right)\end{array}$ & $\begin{array}{c}\mid A F\left(40^{\circ}\right) \\
(\mathrm{dB})\end{array}$ & $\begin{array}{c}\mid A F\left(50^{\circ}\right) \\
(\mathrm{dB})\end{array}$ & $\begin{array}{c}A F\left(70^{\circ}\right) \\
(\mathrm{dB})\end{array}$ \\
\hline GPS & 11.14 & -25 & $9.8^{\circ}$ & -81.5 & -73.8 & -77.7 \\
\hline GA & 10.09 & -17 & $10.6^{\circ}$ & -90.4 & -58.1 & -91.7 \\
\hline Uniform & 12.45 & -13 & $8.1^{o}$ & -35.8 & -25.7 & -31.7 \\
\hline $\begin{array}{c}\text { Dolph- } \\
\text { Chebyshev }\end{array}$ & 11.36 & -25 & $9.6^{\circ}$ & -37.7 & -30.9 & -34.8 \\
\hline $\begin{array}{c}\text { CST } \\
\text { (GPS) }\end{array}$ & 11.14 & -25 & $9.8^{\circ}$ & -43 & -50.5 & -42.9 \\
\hline
\end{tabular}

(a)

\begin{tabular}{|c|c|c|c|c|c|c|}
\hline \multirow{2}{*}{ GPS } & $A_{n}(A)$ & 1.000 & 0.917 & 0.712 & 0.537 & 0.371 \\
\cline { 2 - 7 } & $d_{n}(\lambda)$ & 0.306 & 0.912 & 1.536 & 2.160 & 2.831 \\
\hline \multirow{2}{*}{$\mathrm{GA}$} & $A_{n}(A)$ & 1.000 & 0.705 & 0.686 & 0.722 & 0.362 \\
\cline { 2 - 7 } & $d_{n}(\lambda)$ & 0.270 & 0.806 & 1.343 & 1.911 & 2.474 \\
\hline \multirow{2}{*}{ Uniform } & $A_{n}(A)$ & 1.000 & 1.000 & 1.000 & 1.000 & 1.000 \\
\cline { 2 - 7 } & $d_{n}(\lambda)$ & 0.315 & 0.945 & 1.575 & 2.205 & 2.835 \\
\hline Dolph-Chebyshev & $A_{n}(A)$ & 1.000 & 0.899 & 0.721 & 0.505 & 0.395 \\
\hline
\end{tabular}




\begin{tabular}{|l|l|l|l|l|l|l|}
\hline & $d_{n}(\lambda)$ & 0.315 & 0.945 & 1.575 & 2.205 & 2.835 \\
\hline
\end{tabular}

The second example shows the design of a 24-element array suppressing the average level of the SLL area within the region $\left[6^{\circ}, 90^{\circ}\right]$ while exhibiting a high directivity value. For these requirements, the following fitness function is formed using (5):

Fitness $=20 \log \left\{\int_{\phi_{l}=24^{\circ}}^{\phi_{u}=90^{\circ}} \frac{1}{\Delta \phi_{l}}|A F(\phi)| d \phi\right\}-20 \log D+20 \log \left\{\max \left\{|A F(\phi)|_{\phi_{l}=6^{\circ}}^{\phi_{u}=90^{\circ}}\right\}\right\}$

where broad nulling effects are aimed to be generated within the sub-region $\left[24^{\circ}, 90^{\circ}\right]$. The radiation pattern obtained using GPS algorithm is compared with the designs of uniform, Dolph-Chebyshev, GA algorithm and CST simulator in Fig. 4. It can be observed from the resulted pattern of GPS optimizer that a very broad band $\left[24^{\circ}, 90^{\circ}\right]$ is very successfully achieved with the MSLL of $-58.7 \mathrm{~dB}$. Although the MSLL of GA pattern has the same value with GPS pattern, the level in the broad null desired region $\left[24^{\circ}, 90^{\circ}\right]$ is quite higher than the GPS pattern. Besides, the half power beamwidth and directivity values are also more successfully achieved by using GPS algorithm. As it is observed from Fig. 4, the resulted CST simulation pattern follows the features of GPS pattern except in the broad null region. However, the CST simulation pattern has achieved MSLL of $-36 \mathrm{~dB}$ in broad null region which is more successful than GA pattern. The features of all the pattern types are given in Tablo 2(a) and the corresponding solution spaces of these patterns are given in Tablo 2(b).

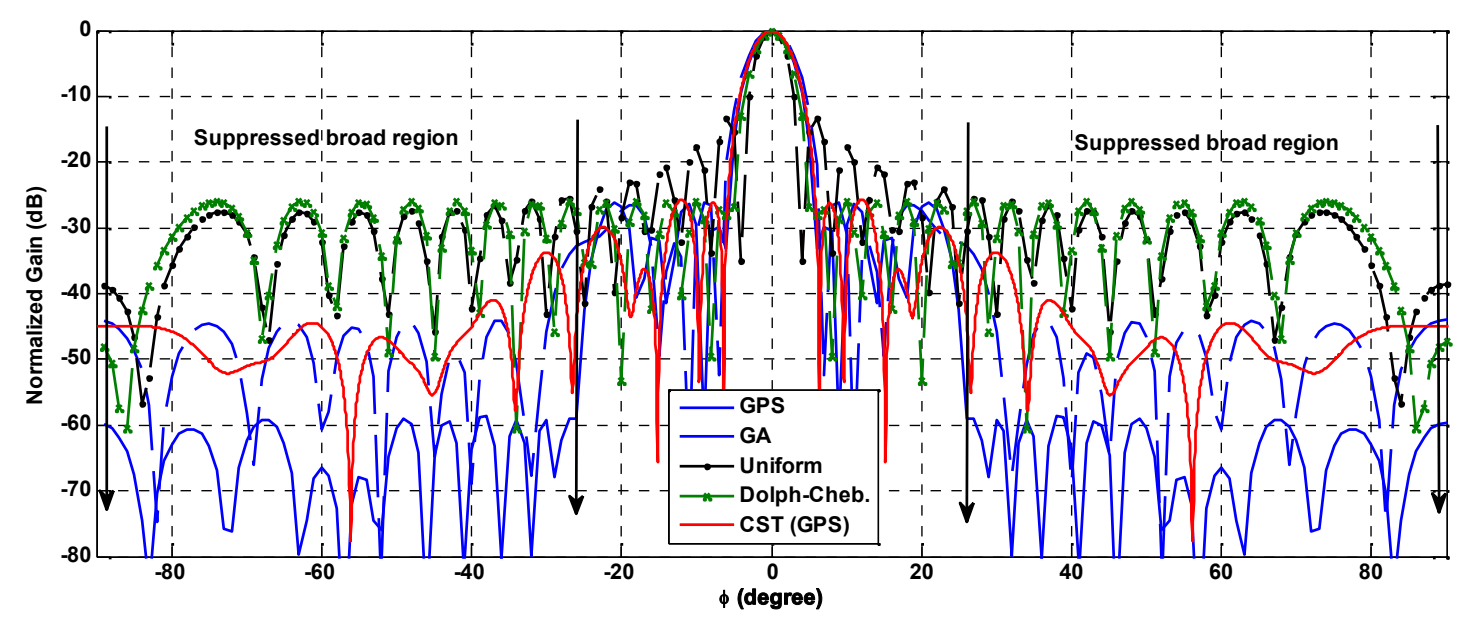

Figure (4): Normalized patterns of the 24-element linear array obtained using five different methods. The region of suppressed SLL is $\left[6^{\circ}, 90^{\circ}\right]$, and required broad nulling effects within the region of $\left[24^{\circ}, 90^{\circ}\right]$ and high directivity, narrow beamwidth are achieved 
Table (2): (a) Features (b) Normalized Solution Spaces, of the Radiation Patterns in Fig.4

\begin{tabular}{|c|c|c|c|c|c|}
\hline $\begin{array}{c}\text { Optimization } \\
\text { Algorithm }\end{array}$ & $\mathrm{D}$ & $\begin{array}{c}\mathrm{MSLL} \\
(\mathrm{dB})\end{array}$ & $\begin{array}{c}\mathrm{HPBW} \\
\left(\phi_{H P B W}\right)\end{array}$ & $\begin{array}{c}|A F(\phi)|_{\text {max }} \\
(d B) \text { for } \\
24^{\circ} \leq \phi \leq 90^{\circ}\end{array}$ & $\begin{array}{c}|A F(\phi)|_{\text {average }} \\
(d B) \text { for } \\
24^{\circ} \leq \phi \leq 90^{\circ}\end{array}$ \\
\hline GPS & 23.09 & -26.1 & $4.64^{\circ}$ & -58.7 & -66.66 \\
\hline GA & 20.45 & -26.0 & $5.27^{\circ}$ & -32.7 & -50.22 \\
\hline Uniform & 28.07 & -13.3 & $3.46^{\circ}$ & -25.5 & -33.81 \\
\hline $\begin{array}{c}\text { Dolph- } \\
\text { Chebyshev }\end{array}$ & 25.62 & -26.0 & $4.16^{\circ}$ & -26.1 & -33.54 \\
\hline $\begin{array}{c}\text { CST } \\
\text { (GPS) }\end{array}$ & 22.36 & -25.7 & $4.92^{\circ}$ & -35.8 & -47.13 \\
\hline
\end{tabular}

(a)

\begin{tabular}{|c|c|c|c|c|c|c|c|c|c|c|c|c|c|}
\hline \multirow{2}{*}{ GPS } & $A_{n}(A)$ & 1.000 & 0.892 & 0.813 & 0.794 & 0.764 & 0.636 & 0.485 & 0.447 & 0.419 & 0.287 & 0.187 & 0.075 \\
\hline & $d_{n}(\lambda)$ & 0.303 & 0.908 & 1.510 & 2.110 & 2.712 & 3.322 & 3.920 & 4.512 & 5.120 & 5.673 & 6.173 & 6.746 \\
\hline \multirow{2}{*}{ GA } & $A_{n}(A)$ & 0.952 & 0.891 & 1.000 & 0.847 & 0.755 & 0.621 & 0.604 & 0.516 & 0.473 & 0.327 & 0.254 & 0.144 \\
\hline & $d_{n}(\lambda)$ & 0.290 & 0.791 & 1.290 & 1.794 & 2.293 & 2.793 & 3.292 & 3.791 & 4.290 & 4.799 & 5.298 & 5.944 \\
\hline \multirow{2}{*}{ Uniform } & $A_{n}(A)$ & 1.000 & 1.000 & 1.000 & 1.000 & 1.000 & 1.000 & 1.000 & 1.000 & 1.000 & 1.000 & 1.000 & 1.000 \\
\hline & $d_{n}(\lambda)$ & 0.293 & 0.880 & 1.467 & 2.054 & 2.641 & 3.228 & 3.815 & 4.402 & 4.989 & 5.576 & 6.163 & 6.750 \\
\hline \multirow{2}{*}{$\begin{array}{l}\text { Dolph- } \\
\text { Chebyshev }\end{array}$} & $A_{n}(A)$ & 1.000 & 0.982 & 0.948 & 0.899 & 0.836 & 0.505 & 0.762 & 0.594 & 0.681 & 0.417 & 0.333 & 0.574 \\
\hline & $d_{n}(\lambda)$ & 0.293 & 0.880 & 1.467 & 2.054 & 2.641 & 3.228 & 3.815 & 4.402 & 4.989 & 5.576 & 6.163 & 6.750 \\
\hline
\end{tabular}

(b)

\section{Conclusions:}

In this work, a multi-objective optimization procedure is put forward for the radiation pattern limitations of antenna arrays and this procedure is followed by the two system variables which are the array geometry and feeding network. All possible linear array configurations are analyzed and a formulation for the array factor and directivities are completed.

In the synthesis stage, the fast and efficient optimization process is generated: (i) PSearch algorithm is employed which is a direct, efficient derivative - free algorithm for searching minima of the functions which are not necessarily differentiable, stochastic, or even continuous. (ii) A fitness function is defined so that the limitations of all the main radiation features of a pattern can be searched, which is successfully applied into antenna array synthesizes with the different multi-objective requirements. Finally, the optimized arrays are observed to outperform uniform arrays and representative synthesizes. 


\section{References:}

[1] K. K. Yan and Y. L. Lu, Sidelobe reduction in array pattern synthesis using genetic algorithm, IEEE Trans. Antennas Propagat., Vol. 45, P. 1117-1122, 1997.

[2] G. K. Mahanti, N. Pathak, and P. Mahanti, Synthesis of Thinned Linear Antenna Arrays With Fixed Sidelobe Level Using Real-Coded Genetic Algorithm, Progress In Electromagnetics Research, PIER 75, P. 319-328, 2007.

[3] V. Murino, A. Trucco, and Carlo S. Regazzoni, Synthesis of Unequally Spaced Arrays by Simulated Annealing, IEEE Transactions on Signal Processing, Vol. 44, No. 1, P. 119-127, January 1996.

[4] M. M. Khodier and C. G. Christodoulou, Sidelobe level and null control using particle swarm optimization, IEEE Trans. Antennas Propagat., Vol. 53, P. 26742679, 2005.

[5] V. Torczon, On the convergence of Pattern Search algorithms, SIAM Journal on optimization, 7, P. 1-25, 1997.

[6] R. S. Elliott, Antenna Theory and Design. Prentice-Hall, 1981.

[7] W. L. Stutzman and G. A. Thiele, Antenna Theory and Design, John Wiley \& Sons, 1998. 